\title{
High-order methods and numerical boundary conditions
}

\author{
Ercília Sousa \\ CMUC, Department of Mathematics, University of Coimbra, 3001-454 Coimbra, Portugal
}

Received 12 January 2006; received in revised form 21 April 2007; accepted 24 April 2007

Available online 6 June 2007

\begin{abstract}
In this paper we present high-order difference schemes for convection diffusion problems. When we apply high-order numerical methods to problems where physical boundary conditions are not periodic there is a need to choose adequate numerical boundary conditions in order to preserve the high-order accuracy. Next to the boundary we do not usually have enough discrete points to apply the high-order scheme and therefore at these nodes we must consider different approximations, named the numerical boundary conditions.

The choice of numerical boundary conditions can influence the overall accuracy of the scheme and most of the times do influence the stability. Here, we discuss which orders of accuracy are reasonable to be considered at the numerical boundary conditions, such that we do not pay a high price in accuracy and stability.
\end{abstract}

(c) 2007 Elsevier B.V. All rights reserved.

Keywords: High-order finite differences; Convection-diffusion; Boundary conditions

\section{Introduction}

In order to compute approximate solutions for evolutionary partial differential equations with either explicit or implicit schemes, it is necessary to use some form of local approximation; local in the sense that solution values at local nodes are used to generate an approximate solution value at a new time level. Explicit methods have the advantage that they are easily extended to multidimensional problems and the local variables are directly determined from previously known quantities.

In finite differences it is usual to try to make the local domain as compact as possible, for instance using only adjacent nodes when updating at a node. The domain of local approximation may need to be large because the degree of the equation is high or it may need to be enlarged to accommodate a higher-order local approximation for a low order differential equation. In either case schemes are usually derived for infinite space domains; when space boundaries occur they prevent such high-order schemes from being applied directly. One method to deal with the

E-mail address: ecs@mat.uc.pt second of these situations, a high-order local approximation to a low-order equation, is simply to use a lower-order scheme immediately near boundaries and use a high-order scheme for the major part of the interior of the domain. Whether this is useful will depend on the nature of the problem being approximated. If interest is centered on dynamics in the interior and on time scales where boundary effects have not propagated to the region of interest, then this will be a reasonable approximation. If the boundary influences the interior quickly, little may be gained by using a high-order scheme to accurately propagate low-order errors from the boundaries to the interior.

Finite difference schemes are some times afflicted with unwanted oscillations. Some of the ways to deal with unwanted oscillations are: the incorporation of extra artificial viscosity terms into the basic difference equations; local adaptative modification of the discrete scheme to force the solution of the modified equations to have the right behaviour or a change from the simple difference scheme formulae to the one based on upwind differencing adjusted to conform to the flow direction in the solution.

Particular examples of alterations in the equations to be solved are the well-known TVD (total variation diminishing) 
schemes [4] and ENO (essentially non-oscillatory) schemes [5] for difference equations and the schemes SUPG (streamline upwind Petrov-Galerkin) [1] and GLS (Galerkin least squares) [8] versions of the finite element method. This kind of schemes are particularly important for a convection equation but also to some extend for a convection diffusion equation $[7,12,14]$, where a major objective can be to inhibit or prevent oscillations.

Simple upwind differencing has been a popular procedure employed over the years in finite difference schemes to reduce oscillations [15]. This idea was also extended to finite element methods (see for instance [2,6]). High-order methods have gained increased attention in recent years [3] and the necessity of a higher-order scheme is many times related to the fact that simple finite difference scheme formulae generally suffer from either severe stability constraints (central differencing) or loss of accuracy through too much numerical diffusion (upwind differencing).

The work described in this paper is focused on a class of high-order schemes, for the convection-diffusion equation which do allow non-physical oscillatory behaviour in the solution. We derive a high-order scheme which reduces the oscillations near sharply varying gradients. This is achieved by using a discretisation that corresponds to an upstream differencing on the convective term. For comparison we use the Quickest (Quadratic Upstream Interpolation for Convective Kinematics with Estimated Streaming Terms) scheme derived by Leonard [11] that also relies in an upstream differencing and present third-order accuracy. This scheme is well known in the literature by its no numerical diffusion and low dispersion.

Finite difference schemes typically consist of replacement of the individual derivative terms in the partial differential equation by a set of discretised approximations (see e.g. Smith [16]). However, different techniques have been suggested for deriving finite differences for the unsteady convection-diffusion equation (see e.g. Kolesnikov and Baker [9], Morton and Sobey [13] and Xu et al. [20]).

Morton and Sobey [13], derived the Lax-Wendroff scheme, due to Lax and Wendroff [10], and the Quickest scheme, due to Leonard [11], using an evolution operator. In this paper we derive high-order schemes using the evolution operator, associated to the convection-diffusion problem, considered in [13].

The major difficulty in using high-order schemes is due to the presence of boundary conditions. Indeed, periodical boundaries greatly simplify the implementation of highorder schemes. However, there are physical models, such as, in ocean modelling, that must take in consideration the boundaries. Usually, in the literature little is said about high-order difference schemes in the presence of non-periodic boundary conditions since in the majority of cases, to provide a modified scheme at a boundary which retains the accuracy of the scheme used in the interior of the domain will be very difficult. For the schemes derived we will discuss which orders of accuracy are reasonable to be considered at the numerical boundary conditions so that, it will still be worthwhile to use a high-order scheme in the interior.

An outline of the paper is as follows. In Section 2 we describe higher-order schemes for the convection-diffusion problem. Global error and truncation error are analyzed. Also in this section, assuming periodic boundary conditions, we derive stability conditions using the von Neumann analysis. Section 3 is devoted to a convectiondiffusion problem with an inflow boundary. Since next to the boundary we do not have enough discrete points to apply the high-order schemes, we consider different numerical boundary conditions. We finish this section with some test problems. Although the order of accuracy of the overall scheme is influenced by the choice of a different scheme near the boundary, in many situations to use a higher-order scheme is still a better choice.

\section{High-order schemes}

Consider the one-dimensional problem with constant velocity $V$ in the positive $x$ direction and constant diffusion with coefficient $D>0$ :

$\frac{\partial u}{\partial t}+V \frac{\partial u}{\partial x}=D \frac{\partial^{2} u}{\partial x^{2}}, \quad t>0, \quad x \in \mathbb{R}$,

subject to the initial condition

$u(x, 0)=u_{0}(x)$

and the boundary condition

$u(x, t)=0 \quad$ as $\quad|x| \rightarrow \infty$.

This initial value problem can be solved in closed form using Fourier transforms in $x$ to obtain the exact solution,

$u(x, t)=\frac{1}{\sqrt{\pi}} \int_{-\infty}^{+\infty} u_{0}(x-V t+2 \sqrt{D t} \xi) \mathrm{e}^{-\xi^{2}} \mathrm{~d} \xi$.

Let us choose a uniform time step $\Delta t$. Applying the result to evolution over one time step, from time $t_{n}$ to $t_{n+1}=t_{n}+\Delta t$ write

$u\left(x, t_{n}+\Delta t\right)=\int_{-\infty}^{+\infty} u\left(\eta, t_{n}\right) G(x-\eta ; \Delta t) \mathrm{d} \eta$,

where the Green's function is given by

$G(z ; \tau)=\frac{1}{\sqrt{D \pi \tau}} \mathrm{e}^{-(z-V \tau)^{2} / 4 D \tau}$.

As showed by Morton and Sobey [13] to derive either finite difference or finite element approximations we substitute an approximation to $u\left(\eta, t_{n}\right)$ in this integral, and exploit the fact that the integration of a global polynomial can be carried out exactly.

\subsection{Finite difference schemes}

Suppose we have approximations $U_{j}^{n}$ to the values $u\left(x_{j}, t_{n}\right)$ at the mesh points 
$x_{j}=j \Delta x, \quad j=0, \pm 1, \pm 2, \ldots$,

where $\Delta x$ denotes the uniform space step. For this set of values we denote $\mathbf{U}^{n}:=\left\{U_{j}^{n}\right\}$ and $p_{j}\left(x ; \mathbf{U}^{n}\right)$ the interpolating polynomial, associated with the points $x_{j}$, through $U_{j}^{n}$ and the values at a certain number of neighboring points. Then finite difference schemes can be generated from evolution of this interpolating approximation by

$U_{j}^{n+1}=\int_{-\infty}^{+\infty} p_{j}\left(\eta ; \mathbf{U}^{n}\right) G\left(x_{j}-\eta ; \Delta t\right) \mathrm{d} \eta$.

If the approximation scheme obtained comes from approximating $\boldsymbol{U}^{n}$ near $x_{j}$ by a polynomial $p_{j}\left(x ; \mathbf{U}^{n}\right)$, of degree $R$,

$p_{j}\left(x ; \mathbf{U}^{n}\right)=\sum_{r=0}^{R} b_{j r}\left(x-x_{j}\right)^{r}$,

then

$U_{j}^{n+1}=\frac{1}{\sqrt{\pi}} \int_{-\infty}^{+\infty} p_{j}\left(x-V \Delta t+2 \sqrt{D \Delta t} \xi ; \mathbf{U}^{n}\right) \mathrm{e}^{-\xi^{2}} \mathrm{~d} \xi$.

When evaluating the previous integral we come across integrals of the form

$a_{r}=\frac{1}{\sqrt{\pi}} \int_{-\infty}^{+\infty} \xi^{r} \mathrm{e}^{-\xi^{2}} \mathrm{~d} \xi$

For $r=0,1,2, \ldots$ the values of the integrals can be obtained using the recurrence relation:

$a_{0}=1, \quad a_{1}=0$

$a_{r}=\frac{1}{2}(r-1) a_{r-2}, \quad r=2, \ldots$

The approximate solution can be written as

$$
\begin{aligned}
U_{j}^{n+1} & =\frac{1}{\sqrt{\pi}} \int_{-\infty}^{+\infty} \sum_{r=0}^{R} b_{j r}(-V \Delta t+2 \sqrt{D \Delta t} \xi)^{r} \mathrm{e}^{-\xi^{2}} \mathrm{~d} \xi \\
& =\sum_{r=0}^{R} b_{j r} \frac{1}{\sqrt{\pi}} \int_{-\infty}^{+\infty} \sum_{n=0}^{r}\left(\begin{array}{l}
r \\
n
\end{array}\right)(-V \Delta t)^{r-n}(2 \sqrt{D \Delta t})^{n} \xi^{n} \mathrm{e}^{-\xi^{2}} \mathrm{~d} \xi \\
& =\sum_{r=0}^{R} b_{j r} \sum_{n=0}^{r}\left(\begin{array}{l}
r \\
n
\end{array}\right)(-V \Delta t)^{r-n}(2 \sqrt{D \Delta t})^{n} a_{n},
\end{aligned}
$$

where $a_{n}$ is defined by (7). We have

$$
\begin{aligned}
U_{j}^{n+1}= & b_{j 0}-b_{j 1} V \Delta t+b_{j 2}\left[V^{2}(\Delta t)^{2}+2 D \Delta t\right] \\
& -b_{j 3}\left[V^{3}(\Delta t)^{3}+6 V D(\Delta t)^{2}\right] \\
& +b_{j 4}\left[V^{4}(\Delta t)^{4}+12 V^{2} D(\Delta t)^{3}+12 D^{2}(\Delta t)^{2}\right] \\
& -b_{j 5}\left[V^{5}(\Delta t)^{5}+20 V^{3}(\Delta t)^{3} D \Delta t+60 V \Delta t D^{2}(\Delta t)^{2}\right] \\
& +\cdots .
\end{aligned}
$$

Within this framework one can obtain different high-order schemes by different interpolations on a uniform mesh. We use the usual central, backward, forward, second difference and fourth difference operators,
$\Delta_{0} U_{j}:=\frac{1}{2}\left(U_{j+1}-U_{j-1}\right), \quad \Delta_{-} U_{j}:=U_{j}-U_{j-1}$,

$\Delta_{+} U_{j}:=U_{j+1}-U_{j}, \quad \delta^{2} U_{j}:=U_{j+1}-2 U_{j}+U_{j-1}, \quad$ and $\delta^{4} U_{j}:=U_{j+2}-4 U_{j+1}+6 U_{j}-4 U_{j-1}+U_{j-2}$

to evaluate the coefficients $b_{j r}$ in terms of the nodal values $\mathbf{U}^{n}$ and obtain high-order schemes.

For the uniform space step $\Delta x$ and time step $\Delta t$ let:

$\mu=\frac{D \Delta t}{(\Delta x)^{2}}, \quad v=\frac{V \Delta t}{\Delta x}$

$v$ is the Courant (CFL) number.

Quadratic interpolation: If we interpolate $U_{j-1}, U_{j}$ and $U_{j+1}$, then

$U_{j}^{n+1}=\left\{1-v \Delta_{0}+\left(\frac{1}{2} v^{2}+\mu\right) \delta^{2}\right\} U_{j}^{n}$.

Cubic interpolation: If we extend $p_{j}\left(x, \mathbf{U}^{n}\right)$ to include a cubic term, that is, using $U_{j-2}$ as well as $U_{j-1}, U_{j}$ and $U_{j+1}$, then $U_{j}^{n+1}=\left\{1-v \Delta_{0}+\left(\frac{1}{2} v^{2}+\mu\right) \delta^{2}+\frac{1}{6} v\left(1-v^{2}-6 \mu\right) \delta^{2} \Delta_{-}\right\} U_{j}^{n}$.

Quartic interpolation: If a quartic interpolant of $U_{j-2}, \ldots, U_{j+2}$ is used then the approximation formula for $U_{j}^{n+1}$ becomes

$$
\begin{aligned}
U_{j}^{n+1}= & \left\{1-v \Delta_{0}+\left(\frac{1}{2} v^{2}+\mu\right) \delta^{2}+\frac{1}{6} v\left(1-v^{2}-6 \mu\right) \delta^{2} \Delta_{-}\right. \\
& \left.+\frac{1}{24}\left[12 \mu^{2}-2 \mu-12 \mu v(1-v)+v\left(1-v^{2}\right)(2-v)\right] \delta^{4}\right\} U_{j}^{n}
\end{aligned}
$$

Quintic approximation: If a quintic interpolant of $U_{j-3}, \ldots, U_{j+2}$ is used then the approximation formula for $U_{j}^{n+1}$ becomes

$$
\begin{aligned}
U_{j}^{n+1}= & \left\{1-v \Delta_{0}+\left(\frac{1}{2} v^{2}+\mu\right) \delta^{2}+\frac{1}{6} v\left(1-v^{2}-6 \mu\right) \delta^{2} \Delta_{-}\right. \\
& +\frac{1}{24}\left[12 \mu^{2}-2 \mu-12 \mu v(1-v)+v\left(1-v^{2}\right)(2-v)\right] \delta^{4} \\
& \left.+\frac{1}{120}\left[-4 v+5\left(v^{3}+6 v \mu\right)-\left(v^{5}+20 v^{3} \mu+60 v \mu^{2}\right)\right] \delta^{4} \Delta_{-}\right\} U_{j}^{n} .
\end{aligned}
$$

Note that the quadratic interpolation and the cubic interpolation are, respectively, the well-known Lax-Wendroff scheme [10] and Quickest scheme [11]. The schemes not yet studied in the literature are those obtained by the quartic interpolation (11) and quintic interpolation (12).

We can also use this framework to generate schemes on non-uniform meshes. The coefficients $b_{j r}$ in (6) are in this case computed by taking into account that polynomial interpolation is done through points not uniformly spaced. This procedure originates difference operators on a nonuniform mesh analogous to the central, backward, forward, second difference and fourth difference. 


\subsection{Global error and truncation error}

The schemes in the previous section can be written in the matricial form,

$\mathbf{U}^{n+1}=A \mathbf{U}^{n}$,

where the matrix $A$ contains the coefficients of the difference formulas. In order to have a matrix of finite dimensions we assume that the boundary conditions are periodic.

For the exact solution, we denote $\mathbf{u}^{n}$ the set $\mathbf{u}^{n}:=$ $\left\{u\left(x_{j}, t_{n}\right)\right\}$. The error $E^{n}=\mathbf{u}^{n}-\mathbf{U}^{n}$ for the set of nodal errors, is given by

$E^{n+1}=A E^{n}+\Delta t T^{n}$

where $T^{n}$ is the truncation error. For any chosen norm for the error, the practical stability requirement is that $\|A\| \leqslant 1$. Then a global error bound is given by

$\left\|E^{n}\right\| \leqslant\left\|E^{0}\right\|+\Delta t \sum_{j=0}^{n-1}\left\|T^{j}\right\| \leqslant\left\|E^{0}\right\|+(n \Delta t) \max _{0 \leqslant j \leqslant n-1}\left\|T^{j}\right\|$.

Since we assume the boundary conditions are periodic, the stability conditions obtained by $\|A\|_{2} \leqslant 1$, where $\|\cdot\|_{2}$ is the two-norm, are equivalent to the stability conditions obtained using the von Neumann Fourier analysis [17].

The following local truncation error of the schemes can be derived using the modified equation method as in [19] or the Peano kernel theorem as in [13].

Quadratic interpolation: (Lax-Wendroff)

$$
\begin{aligned}
\Delta t T_{j}^{n}= & \frac{1}{6} \Delta x^{3} v\left(1-v^{2}-6 \mu\right) U_{x^{3}}^{n}\left(x_{j}\right) \\
& +\frac{1}{24} \Delta x^{4}\left(12 \mu^{2}-2 \mu+3 v^{2}\left(1-v^{2}-4 \mu\right)\right) U_{x^{4}}^{n}\left(x_{j}\right)+\cdots
\end{aligned}
$$

Cubic interpolation: (Quickest)

$$
\begin{aligned}
\Delta t T_{j}^{n}= & \frac{1}{24} \Delta x^{4}\left(12 \mu^{2}-2 \mu-12 \mu v(1-v)+v\left(1-v^{2}\right)\right. \\
& \times(2-v)) U_{x^{4}}^{n}\left(x_{j}\right)+\cdots
\end{aligned}
$$

Quartic interpolation: We have

$$
\begin{aligned}
\Delta t T_{\left.\right|_{x_{j}}}= & \frac{1}{120} \Delta x^{5}\left(-4 v+5\left(v^{3}+6 v \mu\right)\right. \\
& \left.-\left(v^{5}+20 v^{3} \mu+60 v \mu^{2}\right)\right) U_{x^{5}}^{n}+\cdots
\end{aligned}
$$

Quintic interpolation: We have

$$
\begin{aligned}
\Delta t T_{\left.\right|_{x_{j}}}= & \frac{1}{720} \Delta x^{6}\left(-12 v+4\left(v^{2}+2 \mu\right)+15\left(v^{3}+6 v \mu\right)\right. \\
& -5\left(v^{4}+12 v^{2} \mu+12 \mu^{2}\right)-3\left(v^{5}+20 v^{3} \mu+60 v \mu^{2}\right) \\
& \left.+\left(v^{6}+30 v^{4} \mu+120 \mu^{3}+180 \mu^{2} v^{2}\right)\right) U_{x^{6}}^{n}+\cdots
\end{aligned}
$$

On theoretical grounds, over a finite interval of time these estimates are sensitive to the values of $v$ and $\mu$ considered, since $\mu$ and $v$ need not be constant and may vary depending on how $\Delta x$ and $\Delta t$ are related when we refine the mesh. Nevertheless, in general, we expect the quadratic interpolation to be close to $\mathrm{O}\left(\Delta x^{2}\right)$, the cubic interpolation close to
$\mathrm{O}\left(\Delta x^{3}\right)$, the quartic interpolation close to $\mathrm{O}\left(\Delta x^{4}\right)$ and the quintic interpolation should be $\mathrm{O}\left(\Delta x^{5}\right)$.

\subsection{Fourier stability analysis}

Clearly, the von Neumann condition is very important both practically and theoretically. Even for variable coefficient problems it can be applied locally (with local values of the coefficients) and because instability is a local phenomenon, due to the high frequency modes being the most unstable, it gives necessary stability conditions which can often be shown to be sufficient.

The following important points should be noted concerning the von Neumann method of examining stability. The method which is based on Fourier analysis applies only if the coefficients of the linear difference equation are constant. Boundary conditions are neglected by the von Neumann method which applies in theory only to pure initial value problems with periodic initial data. It does however provide necessary conditions for stability of constant coefficient problems regardless of type of boundary conditions.

If we assume periodic boundary conditions the von Neumann analysis is based on the decomposition of the numerical solution into a Fourier series as

$U_{j}^{n}=\sum_{p=-N}^{N} \kappa_{p}^{n} \mathrm{e}^{\mathrm{i} \xi_{p}(\mathrm{j} \Delta x)}$

where $\mathrm{i}=\sqrt{-1}, \kappa_{p}^{n}$ is the amplification factor of the $p$ th harmonic and $\xi_{p}=\frac{p \pi}{N \Delta x}$. The product $\xi_{p} \Delta x$ is often called the phase angle:

$\theta=\xi_{p} \Delta x$

and covers the domain $(-\pi, \pi)$ in steps of $\pi / N$. The region around $\theta=0$ corresponds to the low frequencies while the region $\theta=\pi$ is associated with the high frequencies. In particular, the value $\theta=\pi$ corresponds to the highest frequency resolvable on the mesh, namely the frequency of wavelength $2 \Delta x$.

Considering a single mode, $\kappa^{n} \mathrm{e}^{\mathrm{i} j \theta}$, its time evolution is determined by the same numerical scheme as the complete numerical solution $U_{j}^{n}$. Hence inserting a representation of this form into a numerical scheme we obtain a stability condition by getting an upper bound for the amplification factor, $\kappa$.

The amplification factor is said to satisfy the von Neumann condition if there is a constant $K$ such that

$|\kappa(\xi)| \leqslant 1+K \Delta t \quad \forall \xi \in \mathbb{R}$.

However, for some problems the presence of the arbitrary constant in (19) is too generous for practical purposes, although being adequate for eventual convergence in the limit $\Delta t \rightarrow 0$. In practice, the inequality (19) is substituted by the following stronger condition:

$|\kappa(\xi)| \leqslant 1 \quad \forall \xi \in \mathbb{R}$. 
This has been called practical stability by Richtmyer and Morton [15] or strict stability by other authors. In some cases condition (19) allows numerical modes to grow exponentially in time for finite values of $\Delta t$. Therefore, the practical, or strict, stability condition (20) is recommended in order to prevent numerical modes from growing faster than the physical modes of the differential equation.

Next, we present some results on stability based in the von Neumann analysis for the four schemes.

The result that follows, about the Lax-Wendroff scheme, can be found in various works such as Warming and Hyett [19].

Proposition 1. A necessary and sufficient condition for stability of the Lax-Wendroff scheme (the quadratic interpolation) is

$v^{2}+2 \mu \leqslant 1$.

The Quickest scheme is more complex than the LaxWendroff scheme and consequently so is its von Neumann stability analysis. A necessary stability condition for the Quickest scheme was given by Leonard [11]. In the next lemma we combine this necessary condition with an additional one.

Lemma 2. If the Quickest scheme (cubic interpolation) is stable then

$v^{2}+6 \mu \frac{1-2 v}{3-2 v} \leqslant 1, \quad v^{2}+6 \mu(1-2 v) \geqslant-2 v$.

Proof. The amplification factor for the Quickest scheme is given by

$$
\begin{aligned}
\kappa(\theta)= & 1-\mathrm{i} v \sin \theta-\left(v^{2}+2 \mu\right)(1-\cos \theta)-\frac{v}{3}\left(1-v^{2}-6 \mu\right) \\
& \times\left(1-\mathrm{e}^{-\mathrm{i} \theta}\right)(1-\cos \theta) .
\end{aligned}
$$

The necessary conditions (21) are obtained imposing $|\kappa(\pi)| \leqslant 1$ since for $\theta=\pi$ we have the fundamental frequency that corresponds to the maximum wavelength. The necessary condition given by Leonard [11], the first condition of (21), was obtained from $\kappa(\pi) \geqslant-1$. We have

$\kappa(\pi)=1-2\left(v^{2}+2 \mu\right)-\frac{4 v}{3}\left(1-v^{2}-6 \mu\right)$

and if $|\kappa(\pi)| \leqslant 1$ then

$v^{2}+2 \mu+\frac{2 v}{3}\left(1-v^{2}-6 \mu\right) \leqslant 1$,

$v^{2}+2 \mu+\frac{2 v}{3}\left(1-v^{2}-6 \mu\right) \geqslant 0$.

The conditions of the lemma follow from these inequalities.

Although analytical von Neumann necessary and sufficient stability conditions have not been so far stated in the literature for the Quickest scheme, they have been computed numerically and plotted in papers by Leonard [11] and Morton and Sobey [13]. In the following theorem however we provide the analytical necessary and sufficient conditions for the Quickest scheme.

Theorem 3. Let $\alpha=2 v \mu-(v / 3)\left(1-v^{2}\right), n=\left(2 \mu+v^{2}\right)^{2}-$ $v^{2}+2 \alpha(1-2 v)$ and $d=4 \alpha\left(2 \mu+v^{2}-v-\alpha\right)$. The Quickest scheme is stable if and only if

(a) The condition $-2 \mu+n-d \leqslant 0$ is satisfied;

(b) Let $S=\{(\mu, v): 0 \leqslant n / 2 d \leqslant 1\}$. For $(\mu, v) \in S$, $n^{2} / 4 d \leqslant 2 \mu$.

Proof. Considering the fact that

$1-\cos \theta=2 \sin ^{2}(\theta / 2)$ and

$\sin ^{2} \theta=4 \sin ^{2}(\theta / 2)\left(1-\sin ^{2}(\theta / 2)\right)$,

the modulus of the amplification factor of the Quickest scheme is given by

$$
\begin{aligned}
|\kappa(\theta)|^{2}= & 1-8 \mu \sin ^{2}(\theta / 2)+4\left[\left(2 \mu+v^{2}\right)^{2}-v^{2}+2 \alpha(1-2 v)\right] \\
& \times \sin ^{4}(\theta / 2)-16 \alpha\left(2 \mu+v^{2}-v-\alpha\right) \sin ^{6}(\theta / 2) .
\end{aligned}
$$

Let $s=\sin ^{2}(\theta / 2)$ then

$$
\begin{aligned}
|\kappa(\theta)|^{2}= & 1-8 \mu s+4\left[\left(2 \mu+v^{2}\right)^{2}-v^{2}+2 \alpha(1-2 v)\right] s^{2} \\
& -16 \alpha\left(2 \mu+v^{2}-v-\alpha\right) s^{3} .
\end{aligned}
$$

It follows $|\kappa(\theta)|^{2}=1+4 s \phi(s)$, where for $s \in[0,1]$,

$$
\begin{aligned}
\phi(s)= & -2 \mu+\left[\left(2 \mu+v^{2}\right)^{2}-v^{2}+2 \alpha(1-2 v)\right] s \\
& -4 \alpha\left(2 \mu+v^{2}-v-\alpha\right) s^{2} .
\end{aligned}
$$

The stability condition $|\kappa(\theta)| \leqslant 1 \forall \theta \in \mathbb{R}$, is satisfied if and only if the condition

$\phi(s) \leqslant 0, \quad s \in[0,1]$

is satisfied. To prove this condition it is necessary and sufficient to prove that $\phi(0) \leqslant 0, \phi(1) \leqslant 0$ and that for $s_{*} \in[0,1]$ such that $\phi^{\prime}\left(s_{*}\right)=0$ then $\phi\left(s_{*}\right) \leqslant 0$.

We have that $\phi(0)=-2 \mu$ and it is negative for all $\mu$. The inequality $\phi(1) \leqslant 0$ is true if and only if the condition (a) of the theorem is satisfied. The zero of the function $\phi^{\prime}(s)$ is

$s_{*}=\frac{\left(2 \mu+v^{2}\right)^{2}-v^{2}+2 \alpha(1-2 v)}{8 \alpha\left(2 \mu+v^{2}-v-\alpha\right)}$.

We want to find $\mu$ and $v$ such that $0 \leqslant s_{*} \leqslant 1$ and $\phi\left(s_{*}\right) \leqslant 0$. We have

$\phi\left(s_{*}\right)=-2 \mu+\frac{1}{4} \frac{\left[\left(2 \mu+v^{2}\right)^{2}-v^{2}+2 \alpha(1-2 v)\right]^{2}}{4 \alpha\left(2 \mu+v^{2}-v-\alpha\right)}$.

The condition $\phi\left(s_{*}\right) \leqslant 0$ is verified if and only if

$\frac{\left[\left(2 \mu+v^{2}\right)^{2}-v^{2}+2 \alpha(1-2 v)\right]^{2}}{16 \alpha\left(2 \mu+v^{2}-v-\alpha\right)} \leqslant 2 \mu$.

and this proves the theorem. 

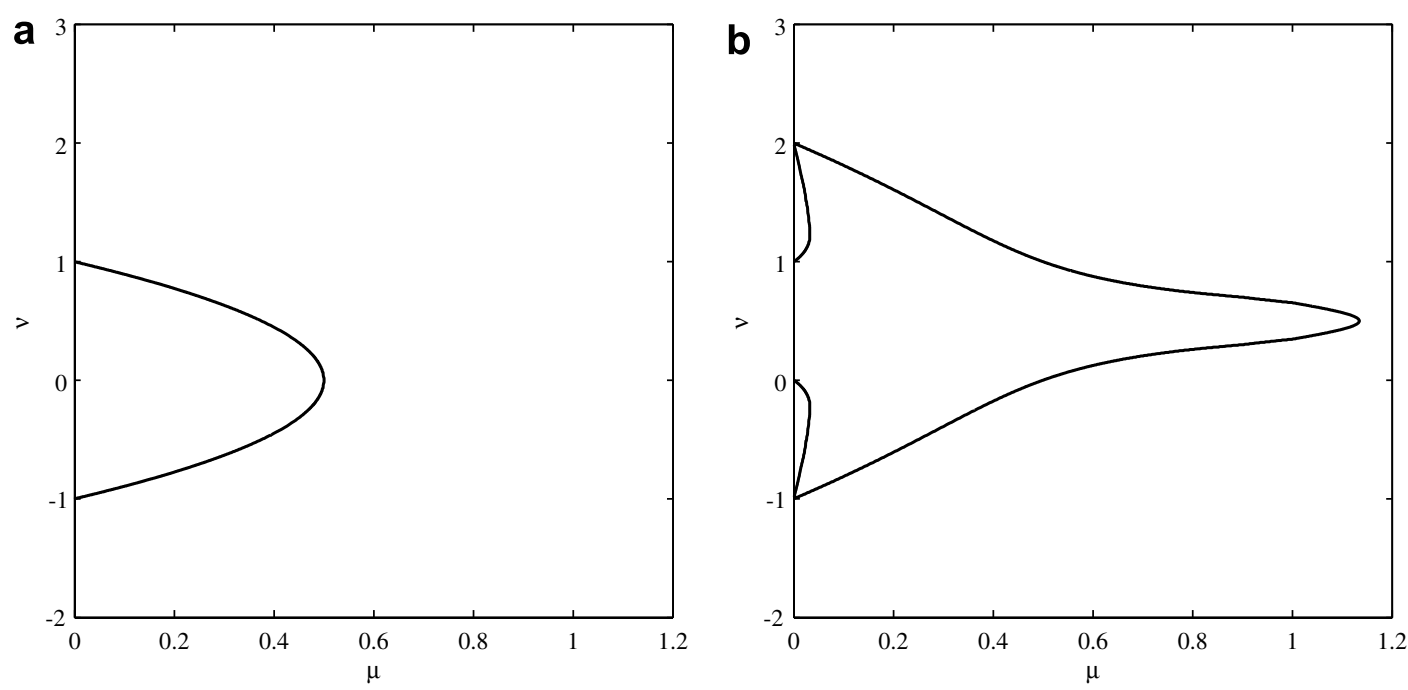

Fig. 1. Fourier stability region for: (a) quadratic and (b) cubic.

We illustrate the stability conditions for the LaxWendroff scheme and Quickest scheme in Fig. 1.

Although the analytical necessary and sufficient conditions presented above, for the Quickest scheme have a cumbersome form, we can check for a fixed value of $v$ or $\mu$ for which values the method is stable. For instance, if $v=1 / 2$ and we want to find for which values of $\mu$ the method is practically stable, we have $\alpha=\mu-1 / 8, n=4(\mu-1 / 8)(\mu-3 / 8)$ and $d=4(\mu-1 / 8)^{2}$. The first condition of the theorem is satisfied for all $\mu$, but the second condition gives us that the method is practically stable for $0 \leqslant \mu \leqslant 9 / 8$. Similarly, if $\mu=0$, then we easily can check that the conditions of the theorem give us $0 \leqslant v \leqslant 1$. Also, for $v=0,1$ we have that the method is stable for $\mu \leqslant 1 / 2$.

All the values obtained by these examples are in agreement with Fig. 1, which was computed numerically.

Now, let us give necessary stability conditions for the schemes obtained by the quartic and quintic interpolations, that we call, respectively, the quartic scheme and the quintic scheme.

\section{Proposition 4. (a) If the Quartic scheme is stable then}

$0 \leqslant 2 \alpha_{2}+4 \alpha_{3}-8 \alpha_{4} \leqslant 1$,

where $\alpha_{2}=\left(v^{2}+2 \mu\right) / 2, \alpha_{3}=v(1-v-6 \mu) / 6$ and $\alpha_{4}=$ $\left(12 \mu^{2}-2 \mu-12 \mu v(1-v)+v\left(1-v^{2}\right)(2-v)\right) / 24$. (b) If the Quintic scheme is stable then

$0 \leqslant 2 \alpha_{2}+4 \alpha_{3}-8 \alpha_{4}-15 \alpha_{5} \leqslant 1$,

where

$\alpha_{5}=\left[-4 v+5\left(v^{3}+6 v \mu\right)-\left(v^{5}+20 v^{3} \mu+60 v \mu^{2}\right)\right] / 120$.

Proof. Let $\theta=\xi \Delta x$. The amplification factor is given by

$$
\begin{aligned}
\kappa(\theta)= & 1-\alpha_{1}\left(\mathrm{e}^{\mathrm{i} \theta}-\mathrm{e}^{-\mathrm{i} \theta}\right)+\alpha_{2}\left(\mathrm{e}^{\mathrm{i} \theta}-2+\mathrm{e}^{-\mathrm{i} \theta}\right)+\alpha_{3}\left(\mathrm{e}^{\mathrm{i} \theta}-3\right. \\
& \left.+3 \mathrm{e}^{-\mathrm{i} \theta}-\mathrm{e}^{-2 \mathrm{i} \theta}\right)+\alpha_{4}\left(\mathrm{e}^{2 \mathrm{i} \theta}-4 \mathrm{e}^{\mathrm{i} \theta}+6-4 \mathrm{e}^{-\mathrm{i} \theta}+\mathrm{e}^{-2 \mathrm{i} \theta}\right) \\
& +\gamma\left(\mathrm{e}^{2 \mathrm{i} \theta}-5 \mathrm{e}^{\mathrm{i} \theta}+10-10 \mathrm{e}^{-\mathrm{i} \theta}+5 \mathrm{e}^{-2 \mathrm{i} \theta}-\mathrm{e}^{-3 \mathrm{i} \theta}\right),
\end{aligned}
$$

where $\alpha_{1}=v / 2, \gamma=0$ for the quartic and $\gamma=\alpha_{5}$ for the quintic. We can write, $\kappa(\theta)=\mathbf{R}(\theta)+\mathbf{i} \mathbf{I}(\theta)$, where

$$
\begin{aligned}
\mathbf{R}(\theta)= & 1-2 \alpha_{2}(1-\cos \theta)-2 \alpha_{3}(\cos \theta-1)^{2} \\
& +4 \alpha_{4}(\cos \theta-1)^{2}+\gamma\left(4\left(1-\cos ^{4} \theta\right)\right. \\
& +15 \cos \theta(\cos \theta-1)) \\
\mathbf{I}(\theta)= & 2 \alpha_{1} \sin \theta+\alpha_{3}(2 \sin \theta(\cos \theta-1)-\sin \theta) \\
& +\gamma\left(4 \sin \theta(\cos \theta-1)^{2}+\sin \theta\right)
\end{aligned}
$$

For $\theta=\pi$, we have $\mathbf{I}(\pi)=0$ and $\mathbf{R}(\pi)=1-4 \alpha_{2}-8 \alpha_{3}+$ $16 \alpha_{4}+30 \gamma$. If the schemes are stable then $|\kappa(\pi)| \leqslant 1$, that is $2 \alpha_{2}+4 \alpha_{3}-8 \alpha_{4}-15 \gamma \geqslant 0, \quad 2 \alpha_{2}+4 \alpha_{3}-8 \alpha_{4}-15 \gamma \leqslant 1$.

(a) For the quartic we have $\gamma=0$ and then $2 \alpha_{2}+4 \alpha_{3}-8 \alpha_{4} \geqslant 0, \quad 2 \alpha_{2}+4 \alpha_{3}-8 \alpha_{4} \leqslant 1$.

(b) For the quintic we have $0 \leqslant 2 \alpha_{2}+4 \alpha_{3}-8 \alpha_{4}-$ $15 \alpha_{5} \leqslant 1$.

Although we have obtained only necessary analytical stability conditions for the quartic and quintic schemes, in Fig. 2, we plot necessary and sufficient conditions computed numerically. We observe from these calculations that for the quartic scheme, the conditions:

$0 \leqslant 2 \alpha_{2}+4 \alpha_{3}-8 \alpha_{4} \leqslant 1$

are necessary and sufficient for stability.

\subsection{Test problem: periodic boundary conditions}

Consider the convection-diffusion problems (1) and (2), for $x \in[0,1]$ and the initial condition $f(x)$ given by

$f(x)=\mathrm{e}^{-(x-0.5)^{2} / L^{2}}, \quad x \in[0,1]$.

The exact solution of this problem can be obtained from the eigenfunctions of the spatial differential operator which are sines and cosines. Hence, the solution is given by means of a Fourier expansion: 

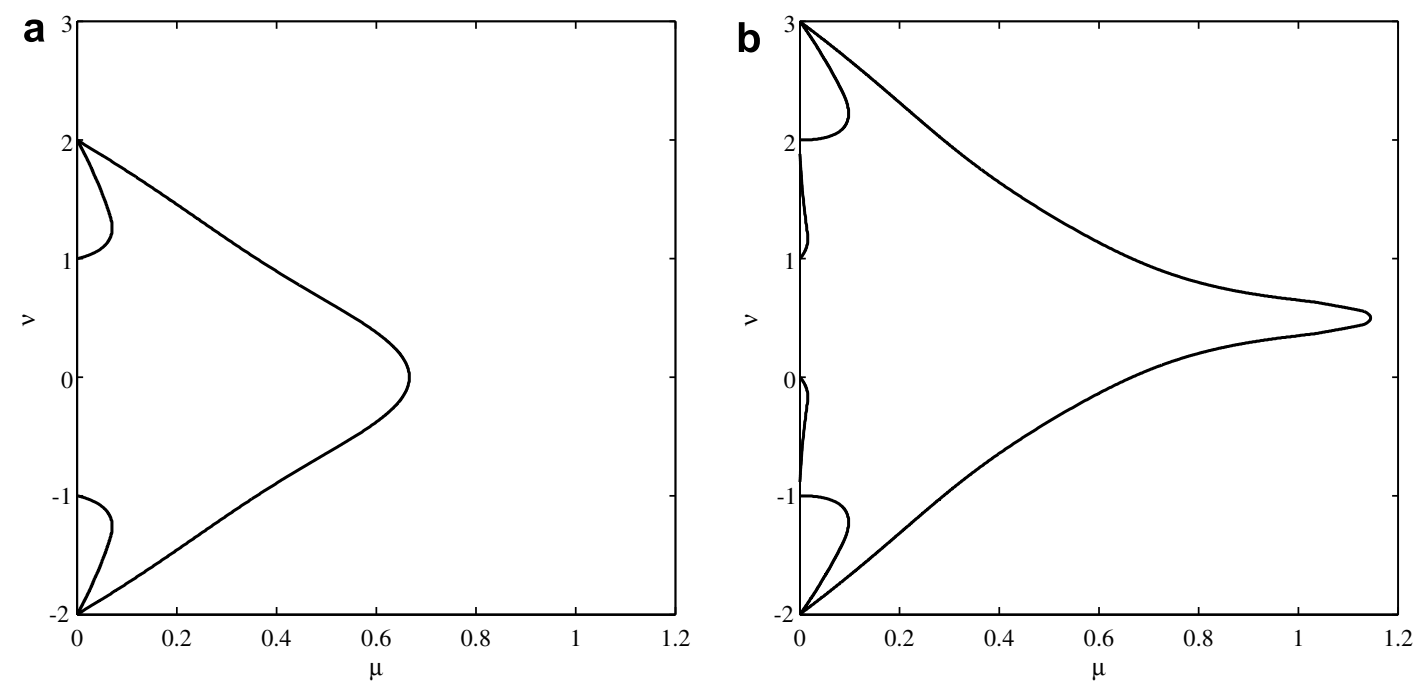

Fig. 2. Fourier stability region for: (a) quartic and (b) quintic.

$u(x, t)=\sum_{k=-\infty}^{+\infty} b_{k} \mathrm{e}^{-4 \pi^{2} k^{2} D t} \mathrm{e}^{2 \pi k(x-V t) i}$

with

$b_{k}=\int_{0}^{1} \mathrm{e}^{-(x-0.5)^{2} / L^{2}} \mathrm{e}^{2 \pi k x i} \mathrm{~d} x$.

Since we are assuming that the boundary conditions are periodic, the stability region for the numerical methods considered are given by the von Neumann stability analysis studied in the previous section and stated in Figs. 1 and 2.

In Fig. 3, we display the exact solution (25) for $L=0.05$ and different instants of time $t=0,0.4,0.8$.

Numerical experiments were conducted for different values of $V$ and $D$ and consequently different Courant numbers $v$ and Péclet numbers $P e$,

$v=\frac{V \Delta t}{\Delta x}, \quad P e=\frac{V \Delta x}{D}$.

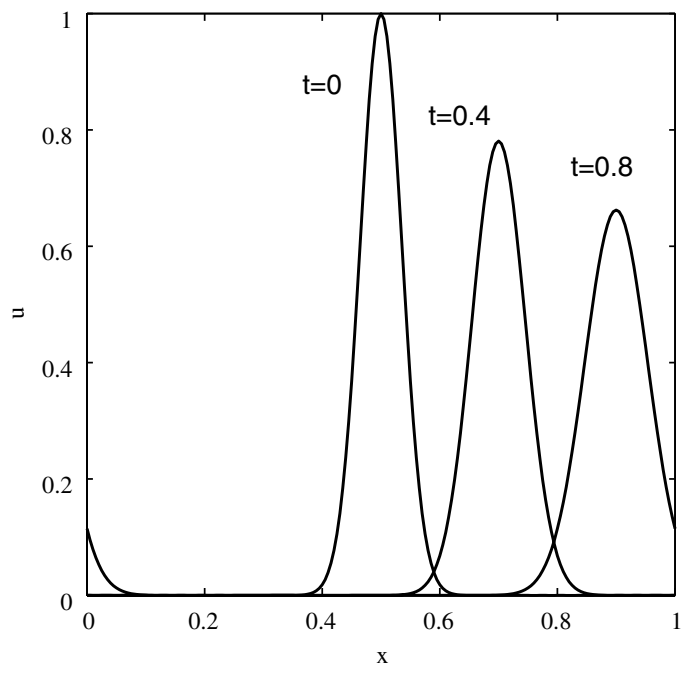

Fig. 3. Exact solution for $t=0,0.4,0.8 ; L=0.05, V=0.5, D=0.001$.
We display in Tables 1 and 2 the global error results for small values of the diffusive parameter $D, D=0.0001$ and $D=0.000001$. The rate of convergence increases from the cubic scheme to the quintic scheme, according to the fact that the schemes are generated by increasing the degree of interpolation.

In Tables 3 and 4 , the diffusive parameter $D$ is closer to the convective parameter $V$ and we consider two different Courant numbers, $v=0.01$ in Table 3 and $v=0.1$ in Table 4. The order of accuracy improves in all methods comparatively to the examples in Tables 1 and 2. Additionally, we observe that the order of accuracy improves as the Courant number increases. Furthermore, the cubic and quartic methods evidences dispersive oscillations that are less present in the quintic scheme (see Fig. 4). These oscillations diminishes as the Courant number increases and completely disappear around $v=0.8$.

Additionally, in Fig. 4 we observe that high-order upwind differencing (quintic scheme) seems to be a better

Table 1

Global $L_{2}$ error of time converged solution for two mesh resolutions at $t=0.8$ for $v=1, V=1, D=0.0001$

\begin{tabular}{llll}
\hline Schemes & $\begin{array}{l}\Delta x=0.01 \\
(\mu=0.01)\end{array}$ & $\begin{array}{l}\Delta x=0.001 \\
(\mu=0.1)\end{array}$ & $\begin{array}{l}\text { Convergence } \\
\text { rate }\end{array}$ \\
\hline Cubic & $0.19531 \times 10^{-2}$ & $0.26399 \times 10^{-4}$ & 1.87 \\
Quartic & $0.10578 \times 10^{-2}$ & $0.14908 \times 10^{-5}$ & 2.85 \\
Quintic & $0.87713 \times 10^{-4}$ & $0.12417 \times 10^{-7}$ & 3.85 \\
\hline
\end{tabular}

$P e=1000 \Delta x$.

Table 2

Global $L_{2}$ error of time converged solution for two mesh resolutions at $t=0.8$ for $v=1, V=1, D=0.000001$

\begin{tabular}{llll}
\hline Schemes & $\begin{array}{l}\Delta x=0.01 \\
(\mu=0.00001)\end{array}$ & $\begin{array}{l}\Delta x=0.001 \\
(\mu=0.0001)\end{array}$ & $\begin{array}{l}\text { Convergence } \\
\text { rate }\end{array}$ \\
\hline Cubic & $0.26944 \times 10^{-4}$ & $0.85748 \times 10^{-6}$ & 1.50 \\
Quartic & $0.15432 \times 10^{-4}$ & $0.53192 \times 10^{-7}$ & 2.48 \\
Quintic & $0.13799 \times 10^{-5}$ & $0.49726 \times 10^{-9}$ & 3.44 \\
\hline
\end{tabular}

$P e=100,000 \Delta x$. 
Table 3

Global $L_{2}$ error of time converged solution for two mesh resolutions at $t=0.8$ for $v=0.01, V=0.5, D=0.001$

\begin{tabular}{llll}
\hline Schemes & $\begin{array}{l}\Delta x=0.01 \\
(\mu=0.002)\end{array}$ & $\begin{array}{l}\Delta x=0.001 \\
(\mu=0.02)\end{array}$ & $\begin{array}{l}\text { Convergence } \\
\text { rate }\end{array}$ \\
\hline Cubic & $0.16248 \times 10^{-1}$ & $0.59929 \times 10^{-4}$ & 2.43 \\
Quartic & $0.33158 \times 10^{-2}$ & $0.92258 \times 10^{-6}$ & 3.56 \\
Quintic & $0.61252 \times 10^{-3}$ & $0.68233 \times 10^{-8}$ & 4.95 \\
\hline
\end{tabular}

$P e=500 \Delta x$.

Table 4

Global $L_{2}$ error of time converged solution for two mesh resolutions at $t=0.8$ for $v=0.1, V=0.5, D=0.001$

\begin{tabular}{llll}
\hline Schemes & $\begin{array}{l}\Delta x=0.01 \\
(\mu=0.02)\end{array}$ & $\begin{array}{l}\Delta x=0.001 \\
(\mu=0.2)\end{array}$ & $\begin{array}{l}\text { Convergence } \\
\text { rate }\end{array}$ \\
\hline Cubic & $0.136805 \times 10^{-1}$ & $0.176549 \times 10^{-4}$ & 2.88 \\
Quartic & $0.285106 \times 10^{-2}$ & $0.105165 \times 10^{-6}$ & 4.43 \\
Quintic & $0.511304 \times 10^{-3}$ & $0.694070 \times 10^{-9}$ & 5.86
\end{tabular}

$P e=500 \Delta x$.

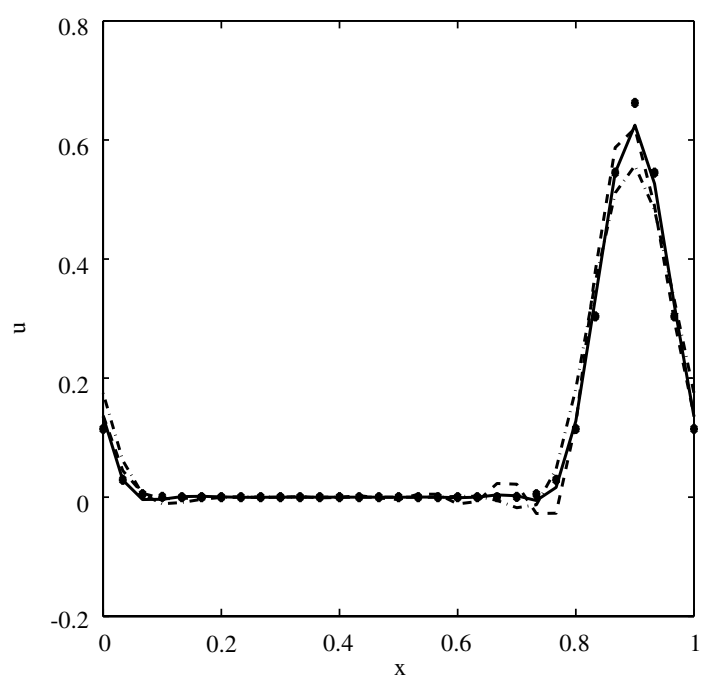

Fig. 4. Solutions for $t=0.8, V=0.5, D=0.001$. Exact $\bullet$; Numerical solutions: cubic (-), quartic (- -), quintic (-); $v=0.1 ; \Delta x=0.03(N=30, N$ number of nodes).

alternative than high-order central differencing (quartic scheme). Although the smooth region accuracy of the fourth-order scheme is good the downstream oscillation problem is actually worse than the third-order. The quintic scheme seems to be almost free of those oscillations. This is due to the fact that we used an upstream differencing that was adjusted to conform to the flow direction. This is better understood if we consider $\mu=0$ in (12) and (18).

The cases where the diffusive parameter $D$ is more dominant are shown in Tables 5 and 6 . The order of accuracy of the cubic and quintic seems to decrease considerably, specially in Table 6 , where the convective parameter is even smaller than in Table 5. The quartic in these cases seems to be a good option. Note that in Table 6 the Courant number was chosen to be $v=0.005$ in order that we can run all schemes inside their stability regions.
Table 5

Global $L_{2}$ error of time converged solution for two mesh resolutions at $t=0.8$ for $v=0.01, V=0.5, D=0.01$

\begin{tabular}{llll}
\hline Schemes & $\begin{array}{l}\Delta x=0.01 \\
(\mu=0.02)\end{array}$ & $\begin{array}{l}\Delta x=0.001 \\
(\mu=0.2)\end{array}$ & $\begin{array}{l}\text { Convergence } \\
\text { rate }\end{array}$ \\
\hline Cubic & $0.33183 \times 10^{-3}$ & $0.44909 \times 10^{-5}$ & 1.87 \\
Quartic & $0.21144 \times 10^{-4}$ & $0.76775 \times 10^{-9}$ & 4.44 \\
Quintic & $0.65020 \times 10^{-6}$ & $0.84342 \times 10^{-10}$ & 3.89 \\
\hline
\end{tabular}

$P e=50 \Delta x$.

Table 6

Global $L_{2}$ error of time converged solution for two mesh resolutions at $t=0.8$ for $v=0.005, V=0.1, D=0.01$

\begin{tabular}{llll}
\hline Schemes & $\begin{array}{l}\Delta x=0.01 \\
(\mu=0.05)\end{array}$ & $\begin{array}{l}\Delta x=0.001 \\
(\mu=0.5)\end{array}$ & $\begin{array}{l}\text { Convergence } \\
\text { rate }\end{array}$ \\
\hline Cubic & $0.47138 \times 10^{-4}$ & $0.46721 \times 10^{-5}$ & 1.00 \\
Quartic & $0.37026 \times 10^{-6}$ & $0.17707 \times 10^{-9}$ & 3.32 \\
Quintic & $0.16133 \times 10^{-6}$ & $0.89163 \times 10^{-10}$ & 3.23 \\
\hline
\end{tabular}

$P e=10 \Delta x$.

In conclusion, the quartic is a good option for the cases where the convective parameter is less dominant, that is, small Péclet numbers and the quintic is by far the best option for big Péclet numbers. These includes small values of $D$, which is the case the convection-diffusion equation approaches the non-diffusive hyperbolic equation.

\section{In the presence of an inflow boundary condition}

Consider the initial boundary condition problem with the convection-diffusion equation (1) defined on the halfline, $x>0$, with the initial condition:

$u(x, 0)=f(x), \quad x>0$

and the boundary conditions

$u(0, t)=g(t), \quad t>0 \quad u(x, t) \rightarrow 0, \quad x \rightarrow \infty$.

We are going to apply the high-order schemes studied earlier to this problem. Therefore we need to consider numerical boundary conditions. In this section we study which numerical boundary conditions are more adequate for the quartic and the quintic approximations.

\subsection{Numerical boundary conditions}

For the quintic approximation we need to consider numerical boundaries at the first two nodes, $j=1$ and $j=2$.

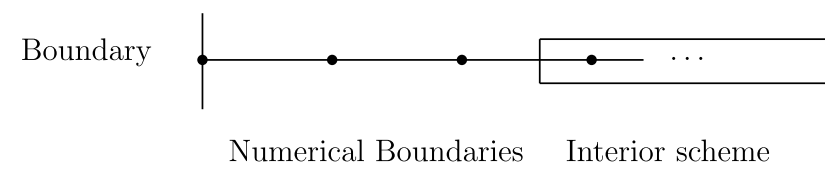

The first idea is to preserve the same degree of interpolation. We derive numerical boundary conditions at $j=1$ and $j=2$ as follows: for $j=1$ we interpolate the mesh 
points $U_{j-1}, \ldots, U_{j+4}$ and for $j=2$ we interpolate the mesh points $U_{j-2}, \ldots, U_{j+3}$. For $j=1$ we get

$$
\begin{aligned}
U_{j}^{n+1}= & \left\{1-v \Delta_{0}+\frac{1}{2}\left(v^{2}+2 \mu\right) \delta^{2}+\frac{1}{6} v\left(1-v^{2}-6 \mu\right) \delta^{2} \Delta_{+}\right. \\
& +\frac{1}{24}\left[-\left(v^{2}+2 \mu\right)-2 v+2 v\left(v^{2}+6 \mu\right)\right. \\
& \left.+\left(v^{4}+12 v^{2} \mu+12 \mu^{2}\right)\right] \delta^{2} \Delta_{+}^{2} \\
& +\frac{1}{120}\left[6 v+5\left(v^{2}+2 \mu\right)-5\left(v^{3}+6 v \mu\right)\right. \\
& -5\left(v^{4}+12 v^{2} \mu+12 \mu^{2}\right) \\
& \left.\left.-\left(v^{5}+20 v^{3} \mu+60 v \mu^{2}\right)\right] \delta^{2} \Delta_{+}^{3}\right\} U_{j}^{n} .
\end{aligned}
$$

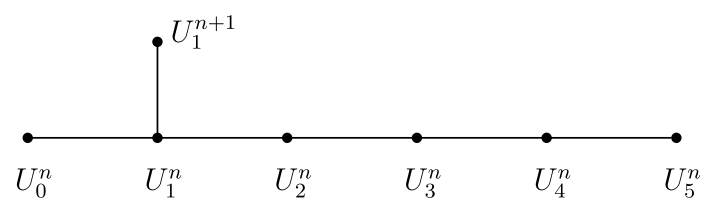

For $j=2$ we have

$$
\begin{aligned}
U_{j}^{n+1}= & \left\{1-v \Delta_{0}+\frac{1}{2}\left(v^{2}+2 \mu\right) \delta^{2}+\frac{v}{6}\left(1-v^{2}-6 \mu\right) \delta^{2} \Delta_{-}\right. \\
& +\frac{1}{24}\left[-\left(v^{2}+2 \mu\right)+2 v-2 v\left(v^{2}+6 \mu\right)\right. \\
& \left.+\left(v^{4}+12 v^{2} \mu+12 \mu^{2}\right)\right] \delta^{4}+\frac{1}{120}\left[-4 v+5\left(v^{3}+6 v \mu\right)\right. \\
& \left.\left.-\left(v^{5}+20 v^{3} \mu+60 v \mu^{2}\right)\right] \delta^{2} \Delta_{-}^{3}\right\} U_{j}^{n} .
\end{aligned}
$$

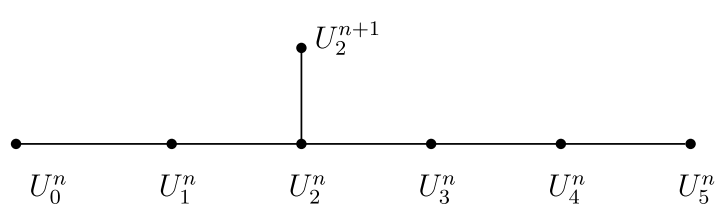

At the first and second nodes, we can also choose lower degrees of interpolation: quadratic, cubic and quartic.

The quadratic interpolation at the first node, $j=1$, leads to the Lax-Wendroff scheme (9).

A cubic downwind interpolation at $j=1$, that interpolates the mesh points $U_{j-1}^{n}, U_{j}^{n}, U_{j+1}^{n}$ and $U_{j+2}^{n}$, is given by $U_{j}^{n+1}=\left\{1-v \Delta_{0}+\left(\frac{1}{2} v^{2}+\mu\right) \delta^{2}+\frac{1}{6} v\left(1-v^{2}-6 \mu\right) \delta^{2} \Delta_{+}\right\} U_{j}^{n}$.

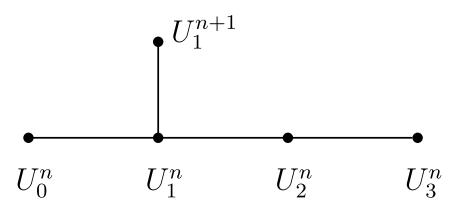

A quartic interpolation at $j=1$, that interpolates the points $U_{j-1}^{n}, U_{j}^{n}, U_{j+1}^{n}$ and $U_{j+2}^{n}$ and $U_{j+3}^{n}$, is given by

$$
\begin{aligned}
U_{j}^{n+1}= & \left\{1-v \Delta_{-}+\frac{1}{2}\left(v^{2}+2 \mu-v\right) \delta^{2}+\frac{1}{6} v\left(1-v^{2}-6 \mu\right) \delta^{2} \Delta_{+}\right. \\
& +\frac{1}{24}\left[-\left(v^{2}+2 \mu\right)-2 v+2 v\left(v^{2}+6 \mu\right)\right. \\
& \left.\left.+\left(v^{4}+12 v^{2} \mu+12 \mu^{2}\right)\right] \delta^{2} \Delta_{+}^{2}\right\} .
\end{aligned}
$$

At the second node, $j=2$, we can consider the quadratic interpolation (9), the cubic interpolation (10) and also the quartic interpolation (11), derived previously.

Let us now describe in detail the schemes we are going to study.

We consider the Quickest scheme (cubic interpolation) with the third-order numerical boundary condition (30) for a self contained study. Note that in [18] it is shown that the Quickest scheme with the third-order numerical boundary condition (30) or with the second-order boundary condition (9) at $j=1$ gives similar accuracy results.

We proceed as follows. We denote cubic $_{3}$ the cubic approximation with the numerical boundary condition (30). For the quartic approximation we use the notation:

quartic $_{j_{1}}, \quad j_{1}=2,3,4$.

The value of $j_{1}$ denotes the numerical boundary condition considered at $j=1$, according to its order of accuracy, that is, $j_{1}=2$ denotes the scheme (9), $j_{1}=3$ denotes the scheme (30) and $j_{1}=4$ denotes the scheme (31).

Similarly, for the quintic approximation we use the notation

quintic $_{j_{1} j_{2}}, \quad j_{1}, j_{2}=2,3,4,5$.

Now, we have an additional numerical boundary condition. The value $j_{1}$ still denotes the boundary condition at the first node, $j=1$, and $j_{2}$ denotes the numerical boundary condition at the second node, $j=2$. For the first node, $j_{1}=2,3,4$ denotes the same as in the quartic scheme and $j_{1}=5$ denotes the scheme (28). For the second node, $j_{2}=2$ denotes the scheme (9), $j_{2}=3$ denotes the scheme (10), $j_{2}=4$ denotes the scheme (11) and $j_{2}=5$ denotes the scheme (29).

\subsection{Global error and stability analysis}

The numerical schemes studied, with the numerical boundary conditions presented, can still be written in the matricial form (13), although the matrix $A$ is now different, taking into account the numerical boundary conditions. Furthermore, we still have the global error given by (14).

In this section, it is very likely that $A$ is a non-normal matrix and therefore the condition $\|A\| \leqslant 1$, is not 
anymore an adequate stability condition as in Section 2.2. We have

$E^{n+1}=A^{n+1} E^{0}+\Delta t \sum_{j=0}^{n} A^{j} T^{n-j}$.

Then a global error bound is given by

$\left\|E^{n+1}\right\| \leqslant\left\|A^{n+1}|||| E^{0}\right\|+(n+1) \Delta t \max _{0 \leqslant j \leqslant n}\left\|A^{j}|||| T^{n-j}\right\|$.

Furthermore, if $\|A\|$ is a matrix such that $\left\|A^{n}\right\| \leqslant K$, for $0<n \Delta t \leqslant T$, then we say that we have Lax stability and

$\left\|E^{n}\right\| \leqslant K|| E^{0}\|+n \Delta t K\| T^{n-1-j} \|$.

For $A$ non-normal, there is usually a transient behaviour of powers before they start to decay exponentially.

For our methods if $\rho(A)>1$ the scheme is unstable. We plot the regions where the schemes are unstable Figs. 5-7. Note that outside the von Neumann stability region, shown

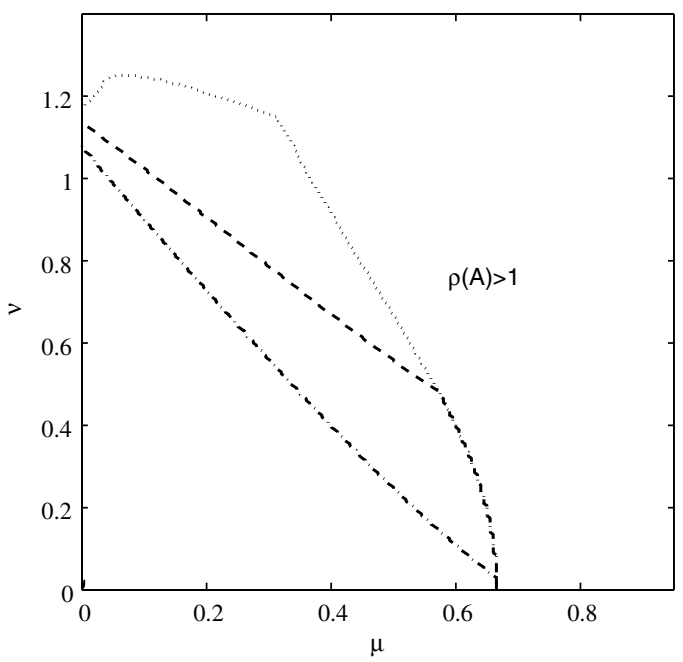

Fig. 5. Quartic approximation with different numerical boundary conditions: quartic $4(-\cdot)$; quartic $_{3}(--)$; quartic $_{2}(\cdots)$.

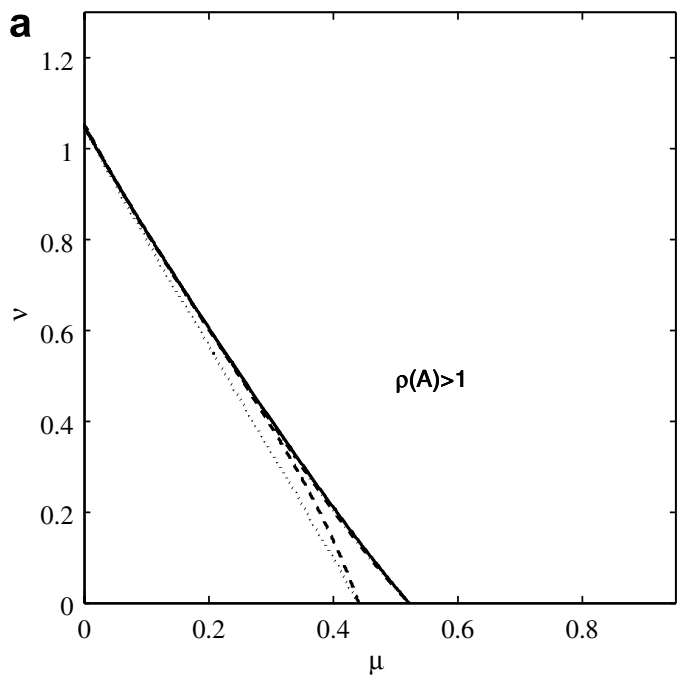

in Figs. 1 and 2, for the interior scheme, the numerical methods with the numerical boundary conditions are still unstable. Therefore, the small part, on the top left of Figs. $5-7$, that is, for small $\mu$ and $v \geqslant 1$, is unstable according to the von Neumann analysis.

\subsection{Test problem: an inflow boundary condition}

If we consider the convection-diffusion problem (1), (26) and (27), then an exact solution of this system on the half line $x \geqslant 0$ is given by

$$
\begin{aligned}
u(x, t)= & \frac{1}{\sqrt{\pi}} \int_{0}^{t} g(t-\tau) G^{*}(x, \tau) \mathrm{d} \tau \\
& +\frac{1}{\sqrt{\pi}} \int_{\frac{V-x}{2 \sqrt{D t}}}^{+\infty} f(x-V t+2 \sqrt{D t} \xi) \mathrm{e}^{-\xi^{2}} \mathrm{~d} \xi \\
& -\frac{1}{\sqrt{\pi}} \int_{\frac{V+x}{2 \sqrt{D t}}}^{+\infty} f(-x-V t+2 \sqrt{D t} \xi) \mathrm{e}^{V x / D} \mathrm{e}^{-\xi^{2}} \mathrm{~d} \xi
\end{aligned}
$$

where the function $G^{*}(x, \tau)$ is given by

$G^{*}(x, \tau)=\frac{x}{2 \sqrt{D} \tau^{2 / 3}} \mathrm{e}^{-(x-V \tau)^{2} / 4 D \tau}$.

Consider the initial data

$u(x, 0)=\mathrm{e}^{-x^{2}}, \quad x>0, \quad u(0, t)=0, \quad t \geqslant 0$.

Our reason for considering this test case is that it is straightforward to calculate an exact solution for this initial profile:

$$
\begin{aligned}
u(x, t)= & \frac{1}{2 \sqrt{4 D t+1}}\left[\mathrm{e}^{-\frac{(x-V t)^{2}}{4 D t+1}} \operatorname{Erfc}\left(-\frac{(x-V t)}{2 \sqrt{D t(4 D t+1)}}\right)\right. \\
& \left.-\mathrm{e}^{-\frac{(x+V t)^{2}}{4 D t+1}}+\frac{V x}{D} \operatorname{Erfc}\left(\frac{(x+V t)}{2 \sqrt{D t(4 D t+1)}}\right)\right],
\end{aligned}
$$

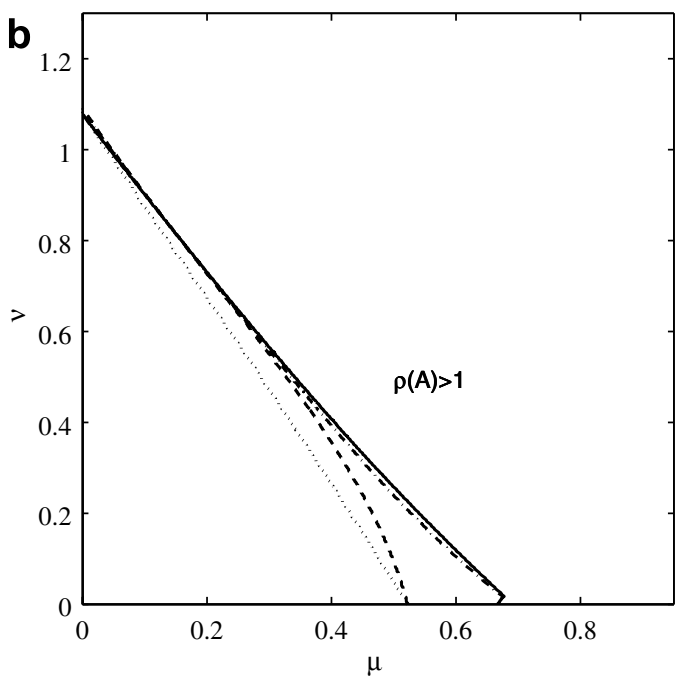

Fig. 6. Quintic approximation with different numerical boundary conditions: (a) quintic $c_{55}(-)$; quintic $c_{54}(-\cdot)$; quintic ${ }_{53}(--)$; quintic $52(\cdots)$. (b) quintic 45 (-); quintic $_{44}(--)$; quintic $43(--)$; quintic $42(\cdots)$. 

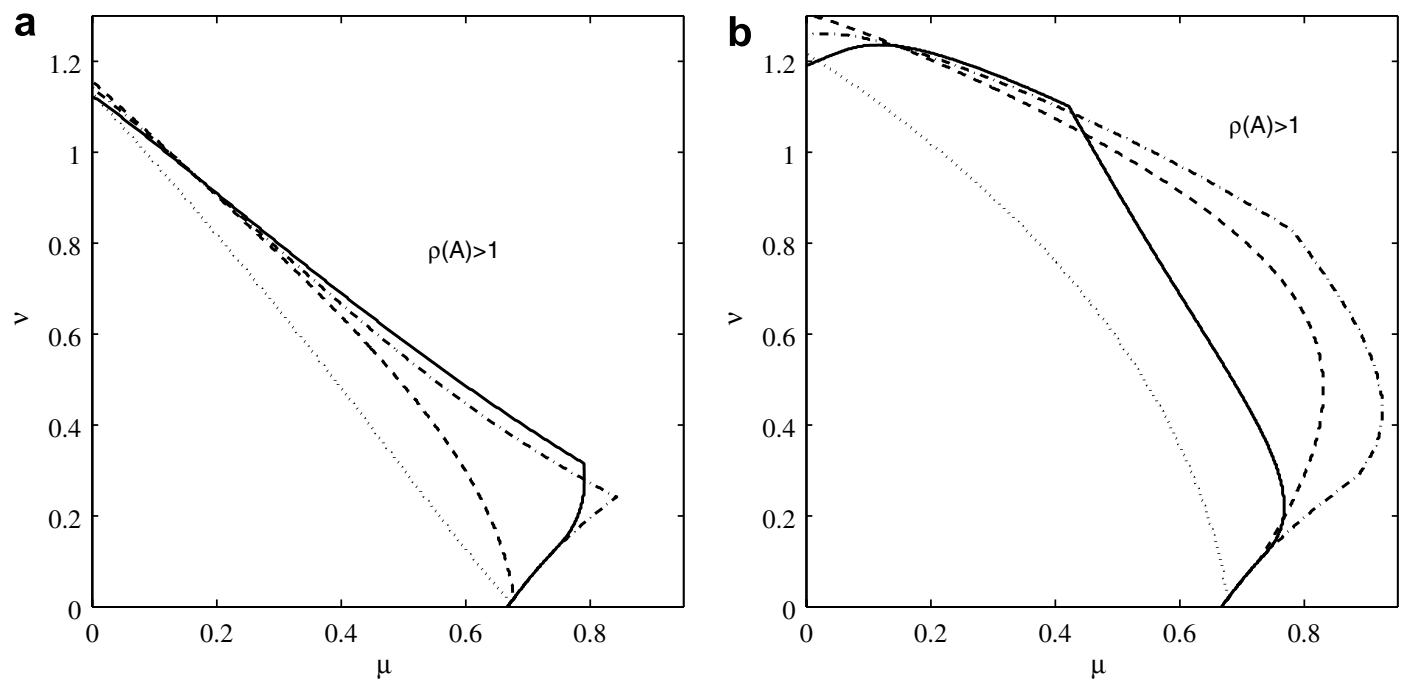

Fig. 7. Quintic approximation with different numerical boundary conditions: (a) quintic $c_{35}(-)$; quintic $c_{34}(-\cdot-)$; quintic 33 (- -); quintic 32 ( . .). (b) quintic $c_{25}(-)$; quintic $_{24}(--)$; quintic $23(--)$; quintic $22(\cdots)$.

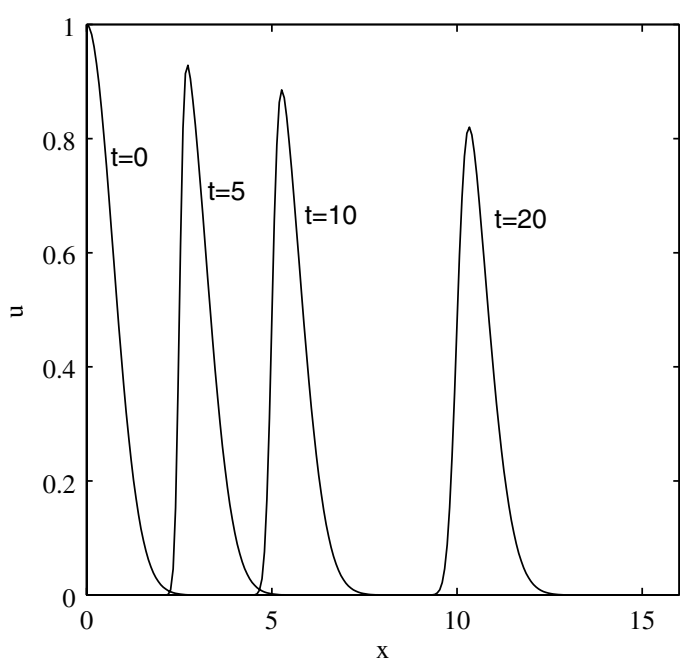

Fig. 8. Exact solution defined by (33) at the times $t=0,5,10,20$, for $V=0.5$ and $D=0.001$.

where $\operatorname{Erfc}(x)=\frac{2}{\sqrt{\pi}} \int_{x}^{\infty} \mathrm{e}^{-s^{2}} \mathrm{~d} s$. The time evolution of the solution is shown in Fig. 8, at the times $t=0,5,10,20$ and for $V=0.5$ and $D=0.001$.

In what follows, for the initial solution $u(x, 0)=\mathrm{e}^{-x^{2}}$, we compute the approximate solutions for a finite domain $0 \leqslant x \leqslant 6$ at $t=5$.

Tables 7 and 8 show the global $L_{2}$ error for the cubic, quartic and quintic with different numerical boundary conditions. In Table 7, we assume $V=0.5, D=0.001$ and in Table $8 V=0.5, D=0.0001$. The convergence in the second case is slower, that is, for the same space step, the error is smaller for $D=0.001$ (Table 7) than for $D=0.0001$ (Table 8). In the later case we have a steepest gradient, as we can see in Fig. 10, and therefore to remove the oscillations is more difficult. Also the differences between Tables 7 and 8 confirms what was observed for the periodic case: the quintic approximation shows to be considerably advantageous over the quartic scheme for small values of $D$.
For the quartic, all the numerical boundary conditions chosen seems to give good results. Note that the quartic 2 performs quite well.

Concerning the quintic, if we take into account both cases, $D=0.001$ (Table 7) and $D=0.0001$ (Table 8), the best results seems to be given by quintic $c_{54}$, quintic 44 (although similar to quintic $c_{45}$ ), and quintic $c_{35}$ (although similar to quintic ${ }_{34}$ ).

In Figs. 9 and 10, we plot results for the cubic $_{3}$, quartic 4 , quintic $_{54}$ and quintic $_{35}$. The quintic presents smaller oscillations and they disappear more quickly as we refine the mesh.

We observe that in Table 7 the quintic with secondorder boundary condition at the first node becomes quite good as we refine the mesh, although this is not an advantage when using high-order schemes, since the main advantage of considering an higher-order scheme is to get more accuracy with less refined meshes.

The extension of this work to higher dimensions is easily achievable. If we consider the two-dimensional convectiondiffusion problem:

$\frac{\partial u}{\partial t}+V \frac{\partial u}{\partial x}+W \frac{\partial u}{\partial y}=D\left(\frac{\partial^{2} u}{\partial x^{2}}+\frac{\partial^{2} u}{\partial y^{2}}\right)$

subject to the initial condition

$u(x, y, 0)=u_{0}(x, y)$

we obtain the two-dimensional evolution operator

$$
\begin{aligned}
u\left(x, y, t_{n+1}\right)= & \frac{1}{\pi} \int_{-\infty}^{+\infty} \int_{-\infty}^{+\infty} u(x-V \Delta t+2 \sqrt{D \Delta t} \xi, y \\
& \left.-W \Delta t+2 \sqrt{D \Delta t} \tau, t_{n}\right) \mathrm{e}^{-\xi^{2}-\tau^{2}} \mathrm{~d} \xi \mathrm{d} \tau
\end{aligned}
$$

Therefore, by approximating $u\left(x, y, t_{n}\right)$ in (36) by a local polynomial of degree $K$ around the point $\left(x_{j}, y_{k}\right)$, namely

$p_{j k}(x, y)=\sum_{r, s=0, r+s \leqslant K}^{K} b_{r s}\left(x-x_{j}\right)^{r}\left(y-y_{k}\right)^{s}$,

we can generate finite difference schemes in two dimensions from the approximation: 
Table 7

Global $L_{2}$ error of time converged solution for three mesh resolutions: $t=5, x \in[0,6], D=0.001, V=0.5, v=0.01$

\begin{tabular}{|c|c|c|c|c|c|}
\hline \multirow[t]{2}{*}{ Schemes } & & \multicolumn{3}{|l|}{ Error $L_{2}$} & \multirow{2}{*}{$\begin{array}{l}\text { Convergence } \\
p\end{array}$} \\
\hline & & $\Delta x=0.1$ & $\Delta x=0.05$ & $\Delta x=0.01$ & \\
\hline cubic & cubic $_{3}$ & $0.2402 \mathrm{E}-00$ & $0.1177 \mathrm{E}-00$ & $0.3802 \mathrm{E}-02$ & 1.80 \\
\hline quartic & $\begin{array}{l}\text { quartic }_{4} \\
\text { quartic }_{3} \\
\text { quartic }_{2}\end{array}$ & $\begin{array}{l}0.2768 \mathrm{E}-00 \\
0.2529 \mathrm{E}-00 \\
0.2317 \mathrm{E}-00\end{array}$ & $\begin{array}{l}0.7074 \mathrm{E}-01 \\
0.7733 \mathrm{E}-01 \\
0.7978 \mathrm{E}-01\end{array}$ & $\begin{array}{l}0.8088 \mathrm{E}-03 \\
0.8099 \mathrm{E}-03 \\
0.8026 \mathrm{E}-03\end{array}$ & $\begin{array}{l}2.53 \\
2.49 \\
2.46\end{array}$ \\
\hline quintic $_{5 n}$ & $\begin{array}{l}\text { quintic }_{55} \\
\text { quintic }_{54} \\
\text { quintic }_{53} \\
\text { quintic }_{52}\end{array}$ & $\begin{array}{l}0.2825 \mathrm{E}-00 \\
0.1174 \mathrm{E}-00 \\
0.1276 \mathrm{E}-00 \\
0.3817 \mathrm{E}-00\end{array}$ & $\begin{array}{l}0.2690 \mathrm{E}-01 \\
0.2195 \mathrm{E}-01 \\
0.2860 \mathrm{E}-01 \\
0.9638 \mathrm{E}-01\end{array}$ & $\begin{array}{l}0.8415 \mathrm{E}-03 \\
0.8417 \mathrm{E}-03 \\
0.8386 \mathrm{E}-03 \\
0.1091 \mathrm{E}-02\end{array}$ & $\begin{array}{l}2.53 \\
2.14 \\
2.18 \\
2.54\end{array}$ \\
\hline quintic $_{4 n}$ & $\begin{array}{l}\text { quintic }_{45} \\
\text { quintic }_{44} \\
\text { quintic }_{43} \\
\text { quintic }_{42}\end{array}$ & $\begin{array}{l}0.1506 \mathrm{E}-00 \\
0.1279 \mathrm{E}-00 \\
0.1283 \mathrm{E}-00 \\
0.3134 \mathrm{E}-00\end{array}$ & $\begin{array}{l}0.1956 \mathrm{E}-01 \\
0.2089 \mathrm{E}-01 \\
0.2687 \mathrm{E}-01 \\
0.7683 \mathrm{E}-01\end{array}$ & $\begin{array}{l}0.8429 \mathrm{E}-03 \\
0.8430 \mathrm{E}-03 \\
0.8407 \mathrm{E}-03 \\
0.9384 \mathrm{E}-03\end{array}$ & $\begin{array}{l}2.25 \\
2.18 \\
2.18 \\
2.52\end{array}$ \\
\hline quintic $_{3 n}$ & $\begin{array}{l}\text { quintic }_{35} \\
\text { quintic }_{34} \\
\text { quintic }_{33} \\
\text { quintic }_{32}\end{array}$ & $\begin{array}{l}0.1331 \mathrm{E}-00 \\
0.1396 \mathrm{E}-00 \\
0.1407 \mathrm{E}-00 \\
0.2396 \mathrm{E}-00\end{array}$ & $\begin{array}{l}0.2425 \mathrm{E}-01 \\
0.2638 \mathrm{E}-01 \\
0.3021 \mathrm{E}-01 \\
0.6033 \mathrm{E}-01\end{array}$ & $\begin{array}{l}0.8378 \mathrm{E}-03 \\
0.8378 \mathrm{E}-03 \\
0.8367 \mathrm{E}-03 \\
0.8034 \mathrm{E}-03\end{array}$ & $\begin{array}{l}2.20 \\
2.22 \\
2.23 \\
2.47\end{array}$ \\
\hline quintic $_{2 n}$ & $\begin{array}{l}\text { quintic }_{25} \\
\text { quintic }_{24} \\
\text { quintic }_{23} \\
\text { quintic }_{22}\end{array}$ & $\begin{array}{l}0.1474 \mathrm{E}-00 \\
0.1531 \mathrm{E}-00 \\
0.1580 \mathrm{E}-00 \\
0.1927 \mathrm{E}-00\end{array}$ & $\begin{array}{l}0.3870 \mathrm{E}-01 \\
0.4007 \mathrm{E}-01 \\
0.4270 \mathrm{E}-01 \\
0.5496 \mathrm{E}-01\end{array}$ & $\begin{array}{l}0.6957 \mathrm{E}-03 \\
0.6946 \mathrm{E}-03 \\
0.6939 \mathrm{E}-03 \\
0.6906 \mathrm{E}-03\end{array}$ & $\begin{array}{l}2.33 \\
2.34 \\
2.36 \\
2.45\end{array}$ \\
\hline
\end{tabular}

Convergence rate $p$ : between $\Delta x=0.1$ and $\Delta x=0.01$.

Table 8

Global $L_{2}$ error of time converged solution for three mesh resolutions: $t=5, x \in[0,6], D=0.0001, V=0.5, v=0.01$

\begin{tabular}{|c|c|c|c|c|c|}
\hline \multirow[t]{2}{*}{ Schemes } & & \multicolumn{3}{|l|}{ Error $L_{2}$} & \multirow{2}{*}{$\begin{array}{l}\text { Convergence } \\
p\end{array}$} \\
\hline & & $\Delta x=0.05$ & $\Delta x=0.025$ & $\Delta x=0.005$ & \\
\hline cubic & cubic $_{3}$ & $0.4225 \mathrm{E}-00$ & $0.3222 \mathrm{E}-00$ & $0.3845 \mathrm{E}-01$ & 1.04 \\
\hline quartic & $\begin{array}{l}\text { quartic }_{4} \\
\text { quartic }_{3} \\
\text { quartic }_{2}\end{array}$ & $\begin{array}{l}0.5304 \mathrm{E}-00 \\
0.4741 \mathrm{E}-00 \\
0.4348 \mathrm{E}-00\end{array}$ & $\begin{array}{l}0.3160 \mathrm{E}-00 \\
0.3121 \mathrm{E}-00 \\
0.2955 \mathrm{E}-00\end{array}$ & $\begin{array}{l}0.5252 \mathrm{E}-02 \\
0.5376 \mathrm{E}-02 \\
0.6430 \mathrm{E}-02\end{array}$ & $\begin{array}{l}2.00 \\
1.95 \\
1.83\end{array}$ \\
\hline quintic $_{5 n}$ & $\begin{array}{l}\text { quintic }_{55} \\
\text { quintic }_{54} \\
\text { quintic }_{53} \\
\text { quintic }_{52}\end{array}$ & $\begin{array}{l}0.6185 \mathrm{E}-00 \\
0.2524 \mathrm{E}-00 \\
0.2708 \mathrm{E}-00 \\
0.5027 \mathrm{E}-00\end{array}$ & $\begin{array}{l}0.2728 \mathrm{E}-00 \\
0.1289 \mathrm{E}-00 \\
0.1397 \mathrm{E}-00 \\
0.2429 \mathrm{E}-00\end{array}$ & $\begin{array}{l}0.1373 \mathrm{E}-02 \\
0.1360 \mathrm{E}-02 \\
0.1298 \mathrm{E}-02 \\
0.3330 \mathrm{E}-02\end{array}$ & $\begin{array}{l}2.65 \\
2.27 \\
2.32 \\
2.18\end{array}$ \\
\hline quintic $_{4 n}$ & $\begin{array}{l}\text { quintic }_{45} \\
\text { quintic }_{44} \\
\text { quintic }_{43} \\
\text { quintic }_{42}\end{array}$ & $\begin{array}{l}0.2566 \mathrm{E}-00 \\
0.2589 \mathrm{E}-00 \\
0.2699 \mathrm{E}-00 \\
0.4298 \mathrm{E}-00\end{array}$ & $\begin{array}{l}0.1249 \mathrm{E}-00 \\
0.1277 \mathrm{E}-00 \\
0.1376 \mathrm{E}-00 \\
0.2071 \mathrm{E}-00\end{array}$ & $\begin{array}{l}0.1360 \mathrm{E}-02 \\
0.1350 \mathrm{E}-02 \\
0.1307 \mathrm{E}-02 \\
0.2401 \mathrm{E}-02\end{array}$ & $\begin{array}{l}2.28 \\
2.28 \\
2.31 \\
2.25\end{array}$ \\
\hline quintic $_{3 n}$ & $\begin{array}{l}\text { quintic }_{35} \\
\text { quintic }_{34} \\
\text { quintic }_{33} \\
\text { quintic }_{32}\end{array}$ & $\begin{array}{l}0.2672 \mathrm{E}-00 \\
0.2743 \mathrm{E}-00 \\
0.2791 \mathrm{E}-00 \\
0.3606 \mathrm{E}-00\end{array}$ & $\begin{array}{l}0.1328 \mathrm{E}-00 \\
0.1358 \mathrm{E}-00 \\
0.1420 \mathrm{E}-00 \\
0.1809 \mathrm{E}-00\end{array}$ & $\begin{array}{l}0.1246 \mathrm{E}-02 \\
0.1241 \mathrm{E}-02 \\
0.1236 \mathrm{E}-02 \\
0.1472 \mathrm{E}-02\end{array}$ & $\begin{array}{l}2.33 \\
2.34 \\
2.35 \\
2.39\end{array}$ \\
\hline quintic $_{2 n}$ & $\begin{array}{l}\text { quintic }_{25} \\
\text { quintic }_{24} \\
\text { quintic }_{23} \\
\text { quintic }_{22}\end{array}$ & $\begin{array}{l}0.2857 \mathrm{E}-00 \\
0.2901 \mathrm{E}-00 \\
0.2942 \mathrm{E}-00 \\
0.2280 \mathrm{E}-00\end{array}$ & $\begin{array}{l}0.1512 \mathrm{E}-00 \\
0.1532 \mathrm{E}-00 \\
0.1567 \mathrm{E}-00 \\
0.1724 \mathrm{E}-00\end{array}$ & $\begin{array}{l}0.1648 \mathrm{E}-02 \\
0.1650 \mathrm{E}-02 \\
0.1702 \mathrm{E}-02 \\
0.1964 \mathrm{E}-02\end{array}$ & $\begin{array}{l}2.24 \\
2.25 \\
2.24 \\
2.06\end{array}$ \\
\hline
\end{tabular}

Convergence rate $p$ : between $\Delta x=0.05$ and $\Delta x=0.005$.

$$
\begin{aligned}
U_{j k}^{n+1}= & \frac{1}{\pi} \sum_{r, s=0, r+s \leqslant K}^{K} b_{r s} \int_{\mathbb{R}^{2}}(-V \Delta t+2 \sqrt{D \Delta t} \xi)^{r} \\
& \times(-W \Delta t+2 \sqrt{D \Delta t} \tau)^{s} \mathrm{e}^{-\xi^{2}-\tau^{2}} \mathrm{~d} \xi \mathrm{d} \tau
\end{aligned}
$$

$U_{j k}^{n}$ denotes the approximations to the values $u\left(x_{j}, y_{k}, t_{n}\right)$ at the mesh points $\left(x_{j}, y_{k}\right)=(j \Delta x, k \Delta y), j, k=0, \pm 1, \pm 2, \ldots$ We can proceed in a similar manner for a three-dimensional problem. 

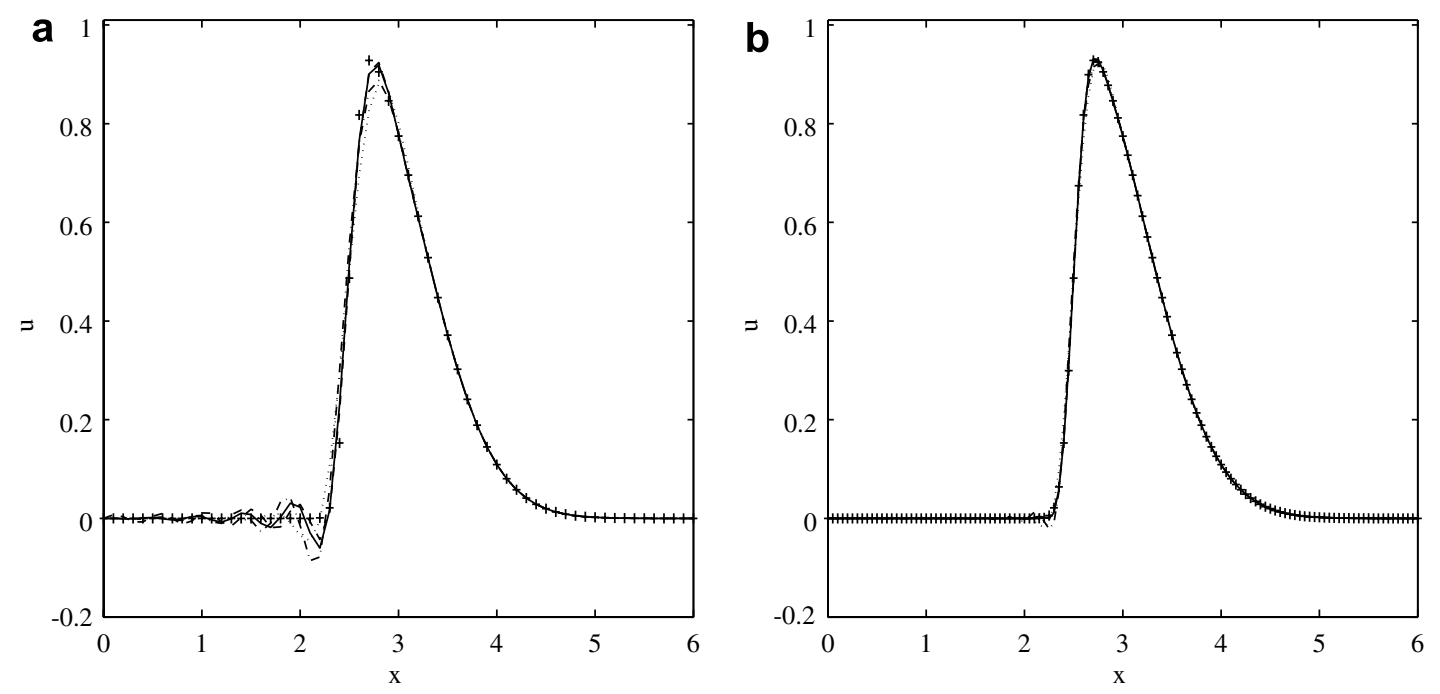

Fig. 9. Solutions for $V=0.5$ and $D=0.001$. Exact solution $(+++)$; cubic $_{3}(\cdots)$; quartic ${ }_{2}(--)$; quintic $54(--)$; quintic $35(-)$. (a) $\Delta x=0.1$; (b) $\Delta x=0.05$.
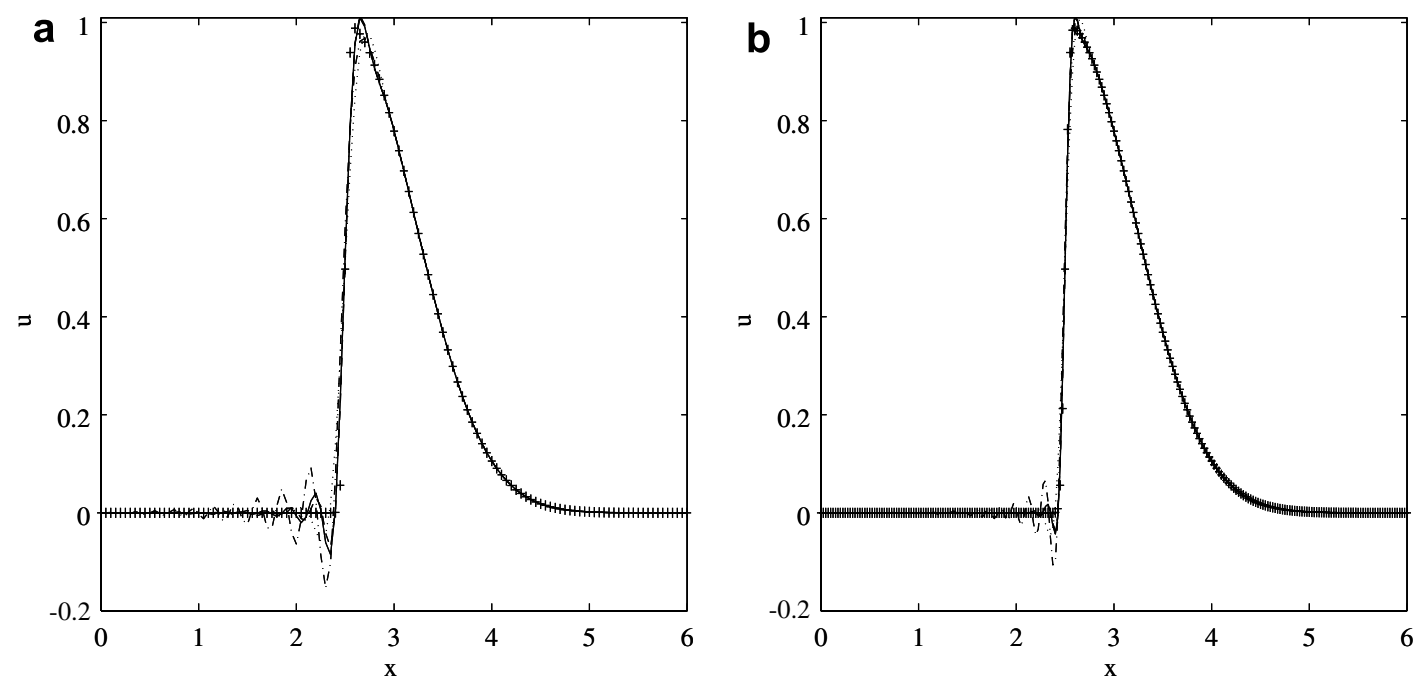

Fig. 10. Solutions for $V=0.5$ and $D=0.0001$. Exact solution $(+++)$; cubic $_{3}(\cdots)$; quartic $2(-\cdot-)$; quintic $54(--)$; quintic 35 (-). (a) $\Delta x=0.05$; (b) $\Delta x=0.025$.

\section{Conclusion}

Schemes of high-order have been developed for the discretisation of the convection-diffusion equation. Firstly, we have derived the schemes for an infinite space domain and have analysed its stability and numerical performance for different values of the convective parameter $V$ and the diffusive parameter $D$ and therefore different Courant numbers and Péclet numbers. The quintic scheme seems to be good in general and, comparatively to the quartic scheme, its best performance is for large Péclet numbers. We also observe that to use a higher-order upstream differencing (the quintic scheme) is a better alternative to a higher-order central scheme (the quartic scheme). The smooth region accuracy for the quartic scheme is good, but the downstream oscillation problem is actually worse than the cubic scheme.
The second part of the paper is devoted to the presence of an inflow boundary. Implementation of boundary conditions can be problematic with such higher-order schemes. Therefore, we present a number of numerical boundary conditions that can be used in order to still benefit from using a higher-order scheme at the interior. According to the numerical results, it seems the quartic scheme performs reasonable well with the numerical boundary conditions of order $\mathrm{O}\left(\Delta x^{2}\right), \mathrm{O}\left(\Delta x^{3}\right)$ and $\mathrm{O}\left(\Delta x^{4}\right)$. For the quintic at the first node, next to the boundary, we can choose a numerical scheme of order at least $\mathrm{O}\left(\Delta x^{3}\right)$ and at the second node we are advised to choose an order not less than $\mathrm{O}\left(\Delta x^{4}\right)$.

This lead us to conjecture that if we have a scheme of higher-order, that is, $\mathrm{O}\left(\Delta x^{n}\right)$, we are advised to choose at the first node, next to the boundary, a scheme of order at least $\mathrm{O}\left(\Delta x^{n-2}\right)$ and on the second node a scheme of order at least $\mathrm{O}\left(\Delta x^{n-1}\right)$. 


\section{References}

[1] A.N. Brooks, T.J.R. Hughes, Streamline upwind Petrov-Galerkin formulations for convection dominated flows with particular emphasis on the incompressible Navier-Stokes equations, Comput. Methods Appl. Mech. Engrg. 54 (1986) 341-355.

[2] I. Christies, D.F. Griffiths, A.R. Mitchell, O.C. Zienkiewicz, Finite element methods for second order differential equations with significant first derivatives, Int. J. Numer. Methods Engrg. 10 (1976) 13891396.

[3] M.O. Deville, P.F. Fischer, E.H. Mund, High-order Methods for Incompressible Fluid Flow, Cambridge University Press, 2002.

[4] A. Harten, High resolution schemes for hyperbolic conservation laws, J. Comput. Phys. 49 (1983) 357-393.

[5] A. Harten, S. Osher, Uniformly high-order accurate non-oscillatory schemes, SIAM J. Numer. Anal. 24 (1987) 279-309.

[6] J.C. Heinrich, P.S. Huyakorn, O.C. Zienkiewicz, an upwind finite element scheme for two-dimensional convective transport equations, Int. J. Numer. Methods Engrg. 11 (1977) 131-143.

[7] J. A Hernandez, High-order finite volume schemes for the advectiondiffusion equation, Int. J. Numer Methods Engrg. 53 (2001) 12111234.

[8] T.J.R. Hughes, L. Franca, G.M. Hulburt, A new finite element formulation for computational fluid dynamics VIII. The Galerkin least squares method for advective-diffusive equations, Comput. Methods Appl. Mech. Engrg. 73 (1989) 173-189.

[9] A. Kolesnikov, A.J. Baker, Efficient implementation of high order methods for the advection-diffusion equation, Comput. Methods Appl. Mech. Engrg. 189 (2000) 701-722.
[10] P.D. Lax, B. Wendroff, Difference schemes for hyperbolic equations with high order of accuracy, Commun. Pure Appl. Math. 17 (1964) 381-398.

[11] B.P. Leonard, A stable and accurate convective modelling procedure based on quadratic upstream interpolation, Comp. Methods Appl. Mech. Engrg. 19 (1979) 59-98.

[12] B.P. Leonard, S. Mokhtari, Beyond first-order upwinding the ultrasharp alternative for non-oscillatory steady-state simulation of convection, Int. J. Numer. Methods Engrg. 30 (1990) 729-766.

[13] K.W. Morton, I.J. Sobey, Discretization of a convection-diffusion equation, IMA J. Numer. Anal. 13 (1993) 141-160.

[14] K.W. Morton, Numerical Solution of Convection-Diffusion Equation Problems, Chapman \& Hall, 1996.

[15] R.D. Richtmyer, K.W. Morton, Difference Methods for Initial-value Problems, Wiley-Interscience, New York, 1967.

[16] G.D. Smith, Numerical Solution of Partial Differential Equations: Finite Difference Methods, Oxford University Press, Oxford, 1985.

[17] G.A. Sod, Numerical Methods in Fluid Dynamics: Initial and Initial Boundary-value Problems, Cambridge University Press, Cambridge, 1988.

[18] E. Sousa, I.J. Sobey, On the influence of numerical boundary conditions, Appl. Numer. Math. 41 (2002) 325-344.

[19] F.F. Warming, B.J. Hyett, The modified equation approach to the stability and accuracy analysis of finite difference methods, J. Comput. Phys. 14 (1974) 159-179.

[20] H.Y. Xu, M.D. Matovic, A. Pollard, Finite difference schemes for three-dimensional time-dependent convection-diffusion equation using full global discretization, J. Comput. Phys. 130 (1997) 109-122. 